\title{
Semistable Reduction in Characteristic Zero for Families of Surfaces and Threefolds
}

\author{
K. Karu \\ Department of Mathematics, Boston University, \\ 111 Cummington, Boston, MA 02215, USA \\ kllkr@math.bu.edu
}

\begin{abstract}
We consider the problem of extending the semistable reduction theorem of [KKMS] from the case of one-parameter families of varieties to families over a base of arbitrary dimension. Following [KKMS], semistable reduction of such families can be reduced to a problem in the combinatorics of polyhedral complexes [AK]. In this paper we solve it in the case when the relative dimension of the morphism is at most three, i.e., for families of surfaces and threefolds.
\end{abstract}

\section{Introduction}

One of the milestones in algebraic geometry is the semistable reduction theorem proved in $[\mathrm{KKMS}]$ :

Theorem 1.1 [KKMS]. Let $f: X \rightarrow C$ be a flat morphism from a variety $X$ onto a nonsingular curve $C$, defined over an algebraically closed field $k$ of characteristic zero. Assume that $0 \in C$ is a point and the restriction $f: X \backslash f^{-1}(0) \rightarrow C \backslash\{0\}$ is smooth. Then there exist a finite morphism $\pi: C^{\prime} \rightarrow C$, with $\pi^{-1}(0)=\left\{0^{\prime}\right\}$, and a proper birational morphism (in fact, a blowup with center lying in the special fiber) $p: X^{\prime} \rightarrow X \times{ }_{C} C^{\prime}$,

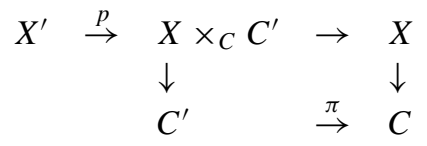

so that the induced morphism $f^{\prime}: X^{\prime} \rightarrow C^{\prime}$ is semistable; i.e.,

(i) both $X^{\prime}$ and $C^{\prime}$ are nonsingular, and

(ii) the special fiber $f^{-1}\left(0^{\prime}\right)$ is a reduced divisor with nonsingular components crossing normally. 
To prove the theorem, Kempf et al. [KKMS] invented the theory of toroidal embeddings and reduced the geometric problem to the following purely combinatorial problem:

Theorem 1.2 [KKMS]. Let $P \subset \mathbb{R}^{n}$ be an $n$-dimensional polytope with vertices lying in the integral points $\mathbb{Z}^{n} \subset \mathbb{R}^{n}$. Then there exists an integer $M$ and a projective subdivision $\left\{P_{\alpha}\right\}_{\alpha}$ of $P$ such that every $P_{\alpha}$ has vertices in $(1 / M) \mathbb{Z}^{n}$ and the volume of $P_{\alpha}$ (in the usual metric) is the minimal possible: $\operatorname{vol}\left(P_{\alpha}\right)=1 / M^{n} n !$.

Here a subdivision is called projective (or coherent) if it is defined by a continuous piecewise linear convex function.

The main goal of $[\mathrm{AK}]$ was to extend the semistable reduction theorem to the case where the base variety has arbitrary dimension. The problem can then be formulated as follows:

Conjecture 1.3. Let $f: X \rightarrow B$ be a surjective morphism of projective varieties with geometrically integral generic fiber, defined over an algebraically closed field of characteristic zero. There exist a proper surjective generically finite morphism $B^{\prime} \rightarrow B$ and a proper birational morphism $X^{\prime} \rightarrow X \times{ }_{B} B^{\prime}$ such that $X^{\prime} \rightarrow B^{\prime}$ is semistable; i.e., for any closed point $x^{\prime} \in X^{\prime}$ and $b^{\prime}=f^{\prime}\left(x^{\prime}\right) \in B^{\prime}$ one can find formal coordinates $x_{1}, \ldots, x_{n}$ at $x^{\prime}$ and $t_{1}, \ldots, t_{m}$ at $b$ so that the morphism $f$ is given by

$$
t_{i}=\prod_{j=l_{i-1}+1}^{l_{i}} x_{j}
$$

for some $0=l_{0}<l_{1}<\cdots<l_{m} \leq n$.

Using the theory of toroidal embeddings, the geometric problem of semistable reduction can again be reduced to a combinatorial problem involving conical polyhedral complexes. The aim of this paper is to solve the combinatorial problem for the case when $f$ has low relative dimension. First, we recall the definition of polyhedral complexes and morphisms.

\subsection{Polyhedral Complexes}

We consider (rational, conical) polyhedral complexes $\Delta=\left(|\Delta|,\left(\left\{\sigma, N_{\sigma}\right\}\right)\right.$ consisting of a finite collection of lattices $N_{\sigma} \cong \mathbb{Z}^{n}$ and rational full cones $\sigma \subset N_{\sigma} \otimes \mathbb{R}$ with a vertex. The cones $\sigma$ are glued together to form the space $|\Delta|$ so that the usual axioms of polyhedral complexes hold:

1. If $\sigma \in \Delta$ is a cone, then every face $\sigma^{\prime}$ of $\sigma$ is also in $\Delta$, and $N_{\sigma^{\prime}}=\left.N_{\sigma}\right|_{\operatorname{Span}\left(\sigma^{\prime}\right)}$.

2. The intersection of two cones $\sigma_{1} \cap \sigma_{2}$ is a face of both of them.

A morphism $f_{\Delta}: \Delta_{X} \rightarrow \Delta_{B}$ of polyhedral complexes $\Delta_{X}=\left(\left|\Delta_{X}\right|,\left\{\sigma, N_{\sigma}\right\}\right)$ and $\Delta_{B}=\left(\left|\Delta_{B}\right|,\left\{\tau, N_{\tau}\right\}\right)$ is a compatible collection of linear maps $f_{\sigma}:\left(\sigma, N_{\sigma}\right) \rightarrow\left(\tau, N_{\tau}\right)$; i.e., if $\sigma^{\prime}$ is a face of $\sigma$, then $f_{\sigma^{\prime}}$ is the restriction of $f_{\sigma}$. We only consider morphisms $f: \Delta_{X} \rightarrow \Delta_{B}$ such that $f_{\sigma}^{-1}(0) \cap \sigma=\{0\}$ for all $\sigma \in \Delta_{X}$. 
Polyhedral complexes arise naturally in the theory of toroidal embeddings [KKMS]. They generalize the notion of fans of toric varieties. An open embedding of varieties $U_{X} \subset X$ is said to be toroidal if it is locally formally isomorphic to a torus embedding $T \subset X_{\sigma}$; a morphism of toroidal embeddings is a morphism of varieties that locally formally comes from a toric morphism. To a toroidal embedding one associates a polyhedral complex, and a morphism of toroidal embeddings gives rise to a morphism of polyhedral complexes. The condition of semistability, when applied to a toroidal embedding, translates into the following condition on the associated morphism of polyhedral complexes.

Definition 1.4. A surjective morphism $f_{\Delta}: \Delta_{X} \rightarrow \Delta_{B}$ such that $f^{-1}(0)=\{0\}$ is semistable if:

1. $\Delta_{X}$ and $\Delta_{B}$ are nonsingular.

2. For any cone $\sigma \in \Delta_{X}$, we have $f(\sigma) \in \Delta_{B}$ and $f\left(N_{\sigma}\right)=N_{f(\sigma)}$.

We say that $f$ is weakly semistable if it satisfies the two conditions except that $\Delta_{X}$ may be singular.

The following two operations are allowed on $\Delta_{X}$ and $\Delta_{B}$ :

1. Projective subdivisions $\Delta_{X}^{\prime}$ of $\Delta_{X}$ and $\Delta_{B}^{\prime}$ of $\Delta_{B}$ such that $f$ induces a morphism $f^{\prime}: \Delta_{X}^{\prime} \rightarrow \Delta_{B}^{\prime}$

2. Lattice alterations: let $\Delta_{X}^{\prime}=\left(\left|\Delta_{X}\right|,\left\{\sigma, N_{\sigma}^{\prime}\right\}\right), \Delta_{B}^{\prime}=\left(\left|\Delta_{B}\right|,\left\{\tau, N_{\tau}^{\prime}\right\}\right)$, for some compatible collection of sublattices $N_{\tau}^{\prime} \subset N_{\tau}, N_{\sigma}^{\prime}=f^{-1}\left(N_{\tau}^{\prime}\right) \cap N_{\sigma}$, and let $f^{\prime}: \Delta_{X}^{\prime} \rightarrow \Delta_{B}^{\prime}$ be the morphism induced by $f$.

Conjecture 1.5 (Combinatorial Semistable Reduction). Given a surjective morphism $f: \Delta_{X} \rightarrow \Delta_{B}$, such that $f^{-1}(0)=\{0\}$, there exists a projective subdivision $f^{\prime}: \Delta_{X}^{\prime} \rightarrow$ $\Delta_{B}^{\prime}$ followed by a lattice alteration $f^{\prime \prime}: \Delta_{X}^{\prime \prime} \rightarrow \Delta_{B}^{\prime \prime}$ so that $f^{\prime \prime}$ is semistable.

$$
\begin{array}{lllll}
\Delta_{X^{\prime \prime}} & \rightarrow & \Delta_{X^{\prime}} & \rightarrow & \Delta_{X} \\
\downarrow f^{\prime \prime} & & \downarrow f^{\prime} & & \downarrow f \\
\Delta_{B^{\prime \prime}} & \rightarrow & \Delta_{B^{\prime}} & \rightarrow & \Delta_{B}
\end{array}
$$

The importance of Conjecture 1.5 lies in the fact that it implies Conjecture 1.3 [AK]. Although we are concerned with the combinatorial version of semistable reduction in this paper, we indicate briefly how the two conjectures are related. It is shown in $[\mathrm{AK}]$ that a morphism $f: X \rightarrow B$ as in Conjecture 1.3 can be modified to a toroidal morphism, and so we get a morphism of polyhedral complexes $f_{\Delta}: \Delta_{X} \rightarrow \Delta_{B}$. Then one checks that if $f_{\Delta}$ is semistable according to Definition 1.4, then $f$ is semistable as defined in Conjecture 1.3. It remains to show that the two combinatorial operations on $f_{\Delta}: \Delta_{X} \rightarrow \Delta_{B}$ have geometric analogues for $f: X \rightarrow B$. Indeed, subdivisions of $\Delta_{X}$ and $\Delta_{B}$ correspond to birational morphisms (see [KKMS]), and a lattice alteration corresponds to a finite base change (see $[\mathrm{AK}]$ ).

In the case when $\operatorname{dim}\left(\Delta_{B}\right)=1$, Conjecture 1.5 reduces to the combinatorial version of the semistable reduction theorem proved in [KKMS]. In [AK] the conjecture was proved with semistable replaced by weakly semistable. The main result of this paper is 
Theorem 1.6. Conjecture 1.5 is true if $f_{\Delta}$ has relative dimension $\leq 3$. Hence, Conjecture 1.3 is true if $f$ has relative dimension $\leq 3$.

The relative dimension of a linear map $f_{\sigma}: \sigma \rightarrow \tau$ of cones $\sigma, \tau$ is $\operatorname{dim}(\sigma)-$ $\operatorname{dim}(f(\sigma))$. The relative dimension of $f_{\Delta}: \Delta_{X} \rightarrow \Delta_{B}$ is by definition the maximum of the relative dimensions of $f_{\sigma}: \sigma \rightarrow \tau$ over all $\sigma \in \Delta_{X}$. To see that the second statement of the theorem follows from the first, consider a surjective morphism of affine toric varieties $f: X_{\sigma} \rightarrow X_{\tau}$ defined by a linear map of cones and lattices $f_{\Delta}:\left(\sigma, N_{\sigma}\right) \rightarrow\left(\tau, N_{\tau}\right)$. A general fiber of this morphism has dimension equal to the rank of the kernel of $f_{\Delta}: N_{\sigma} \rightarrow N_{\tau}$, and this is at least the relative dimension of $f_{\Delta}: \sigma \rightarrow \tau$. Therefore, if a toroidal morphism $f: X \rightarrow B$ has relative dimension $\leq d$, then the associated morphism of polyhedral complexes $f_{\Delta}: \Delta_{X} \rightarrow \Delta_{B}$ also has relative dimension $\leq d$.

We remark that semistable reduction for families of curves over a base of an arbitrary dimension was proved by de Jong $[\mathrm{dJ}]$. Thus, the new result of Theorem 1.6 is semistable reduction for families of surfaces and threefolds.

The rest of the paper is organized as follows. In Section 2 we use the construction of [KKMS] to make $f$ semistable over the edges of $\Delta_{B}$ without increasing the multiplicity of $\Delta_{X}$. In Section 3 we modify the barycentric subdivision of $\Delta_{X}$ so that we get a morphism to the barycentric subdivision of $\Delta_{B}$. It is shown in Section 4 that in certain situations we can choose a modified barycentric subdivision that decreases the multiplicity of $\Delta_{X}$. The conditions when this happens are then verified for relative dimension $\leq 3$ in Section 5 .

\section{Notation and Preliminaries}

\subsection{Notation}

We use notation from [KKMS] and [F]. For a cone $\sigma \in N \otimes \mathbb{R}$ we write $\sigma=\left\langle v_{1}, \ldots, v_{n}\right\rangle$ if the points $v_{1}, \ldots, v_{n}$ lie on the one-dimensional edges of $\sigma$ and generate the cone. If $v_{i}$ are the first lattice points along the edges we call them primitive points of $\sigma$. An $n$-dimensional cone is simplicial if it has exactly $n$ primitive points. For a simplicial cone $\sigma$ with primitive points $v_{1}, \ldots, v_{n}$, the multiplicity of $\sigma$ is

$$
m\left(\sigma, N_{\sigma}\right)=\left[N_{\sigma}: \mathbb{Z} v_{1} \oplus \cdots \oplus \mathbb{Z} v_{n}\right] .
$$

A polyhedral complex $\Delta$ is nonsingular if and only if $m\left(\sigma, N_{\sigma}\right)=1$ for all $\sigma \in \Delta$. To compute the multiplicity of $\sigma$ we can count the representatives $w \in N_{\sigma}$ of classes of $N_{\sigma} / \mathbb{Z} v_{1} \oplus \ldots \oplus \mathbb{Z} v_{n}$ of the form

$$
w=\sum_{i} \alpha_{i} v_{i}, \quad 0 \leq \alpha_{i}<1 .
$$

The set of all such points is denoted by $W(\sigma)$. For cones $\sigma_{1}, \sigma_{2} \in \Delta$ we write $\sigma_{1} \leq \sigma_{2}$ if $\sigma_{1}$ is a face of $\sigma_{2}$. Notice that if $\sigma_{1} \leq \sigma_{2}$, then the multiplicity of $\sigma_{1}$ is at most the multiplicity of $\sigma_{2}$. Hence, to compute the multiplicity of a polyhedral complex $\Delta$, it suffices to consider maximal cones only.

Let $f_{\Delta}: \Delta_{X} \rightarrow \Delta_{B}$ be a morphism of polyhedral complexes, and assume that $\Delta_{B}$ is simplicial. Let $u_{1}, \ldots, u_{m}$ be the primitive points of $\Delta_{B}$, and let $M_{1}, \ldots, M_{m}$ be 
positive integers. By taking the $\left(M_{1}, \ldots, M_{m}\right)$-sublattice at $u_{1}, \ldots, u_{m}$ we mean the lattice alteration $N_{\tau}^{\prime}=\mathbb{Z}\left\{m_{i_{1}} u_{i_{1}}, \ldots, m_{i_{l}} u_{i_{l}}\right\}$ for all cones $\tau \in \Delta_{B}$ with primitive points $u_{i_{1}}, \ldots, u_{i_{l}}$.

A subdivision $\Delta^{\prime}$ of $\Delta$ is called projective if there exists a homogeneous continuous piecewise linear function $\psi:|\Delta| \rightarrow \mathbb{R}$, convex on each cone $\sigma \in \Delta$, and taking rational values on the lattice points $N_{\sigma}$ such that the maximal cones of $\Delta^{\prime}$ are exactly the maximal pieces in which $\psi$ is linear.

\subsection{Applying the Result of $[K K M S]$}

Let $\sigma_{1} \subset \mathbb{R}^{n_{1}}$ and $\sigma_{2} \subset \mathbb{R}^{n_{2}}$ be two cones. We consider $\sigma_{1} \times \sigma_{2}$ as a cone in $\mathbb{R}^{n_{1}+n_{2}}$. If $\left\{\sigma_{1, \alpha}\right\}_{\alpha}$ is a subdivision of $\sigma_{1}$, and $\left\{\sigma_{2, \beta}\right\}_{\beta}$ is a subdivision of $\sigma_{2}$, then $\left\{\sigma_{1, \alpha} \times \sigma_{2, \beta}\right\}_{\alpha, \beta}$ gives us a subdivision of $\sigma_{1} \times \sigma_{2}$.

If $\Delta_{X}$ and $\Delta_{B}$ are simplicial, we say that $f: \Delta_{X} \rightarrow \Delta_{B}$ is simplicial if $f(\sigma) \in \Delta_{B}$ for all $\sigma \in \Delta_{X}$. Assume that $f_{\Delta}: \Delta_{X} \rightarrow \Delta_{B}$ is a simplicial map of simplicial complexes. Let $u_{i}, i=1, \ldots, m$, be the primitive points of $\Delta_{B}$, and let $v_{i j}, i=1, \ldots, m, j=1, \ldots, J_{i}$, be the primitive points of $\Delta_{X}$ such that $v_{i j}$ is mapped to an integer multiple of $u_{i}$. For each $i=1, \ldots, m$ we denote by $\Delta_{X, i}$ the subcomplex of $\Delta_{X}$ lying over the cone $\left\langle u_{i}\right\rangle$ of $\Delta_{B}$ :

$$
\Delta_{X, i}=f_{\Delta}^{-1}\left(\left\langle u_{i}\right\rangle\right)
$$

Note that if we forget the lattices of $\Delta_{X}$, then by the assumption that $f_{\Delta}^{-1}(0)=\{0\}$ we get that $\Delta_{X}=\Delta_{X, 1} \times \cdots \times \Delta_{X, m}$. If $\Delta_{X, i}^{\prime}$ are subdivisions of $\Delta_{X, i}$, we get a subdivison $\Delta_{X}^{\prime}$ of $\Delta_{X}$ by taking the product

$$
\Delta_{X}^{\prime}=\Delta_{X, 1}^{\prime} \times \cdots \times \Delta_{X, m}^{\prime}
$$

Lemma 2.1. If $\Delta_{X, i}^{\prime}$ are projective subdivisions of $\Delta_{X, i}$, then $\Delta_{X}^{\prime}$ is a projective subdivision of $\Delta_{X}$.

Proof. Let $\psi_{i}$ be a convex piecewise linear function defining the subdivison $\left|\Delta_{X, i}^{\prime}\right|$. Extend $\psi_{i}$ linearly to the entire $\left|\Delta_{X}\right|$ by setting $\psi_{i}\left(\left|\Delta_{X, j}\right|\right)=0$ for $j \neq i$. Clearly, $\psi=\sum_{i} \psi_{i}$ is a convex piecewise linear function defining the subdivision $\Delta_{X}^{\prime}$.

Consider the restriction $\left.f_{\Delta}\right|_{\Delta_{X, i}}: \Delta_{X, i} \rightarrow \mathbb{R}_{+} u_{i}$. By the Main Theorem of Chapter 2 in [KKMS] there exist a subdivision $\Delta_{X, i}^{\prime}$ of $\Delta_{X, i}$ and a positive integer $M_{i}$ such that after taking the $M_{i}$-sublattice at $u_{i}$ the induced morphism $\left.f_{\Delta}^{\prime}\right|_{\Delta_{X, i}^{\prime}}$ is semistable. We let $\Delta_{X}^{\prime}$ be the product of the subdivisions $\Delta_{X, i}^{\prime}$, and we take the $\left(M_{1}, \ldots, M_{m}\right)$-sublattice at $\left(u_{1}, \ldots, u_{n}\right)$. Then $f_{\Delta}^{\prime}: \Delta_{X}^{\prime} \rightarrow \Delta_{B}^{\prime}$ is a simplicial map and $\left.f_{\Delta}^{\prime}\right|_{\Delta_{X, i}^{\prime}}$ is semistable for all $i$.

Lemma 2.2. The multiplicity of $\Delta_{X}^{\prime}$ is not greater than the multiplicity of $\Delta_{X}$.

Proof. Let $\sigma \in \Delta_{X}$ have primitive points $v_{i j}$ and let $\sigma^{\prime} \subset \sigma$ be a maximal cone in the subdivision with primitive points $v_{i j}^{\prime}$. The multiplicity of $\sigma^{\prime}$ is the number of points in 
$W\left(\sigma^{\prime}\right)$. We show that $W\left(\sigma^{\prime}\right)$ can be mapped injectively to $W(\sigma)$, hence the multiplicity of $\sigma^{\prime}$ is not greater than the multiplicity of $\sigma$.

If $w^{\prime} \in W\left(\sigma^{\prime}\right)$, we write

$$
w^{\prime}=\sum_{i, j}\left(\beta_{i j}+b_{i j}\right) v_{i j}, \quad 0 \leq \beta_{i j}<1, \quad b_{i j} \in \mathbb{Z}_{+} .
$$

Then $w=\sum_{i j} \beta_{i j} v_{i j} \in N_{\sigma}$ is in $W(\sigma)$. If two points $w_{1}^{\prime}, w_{2}^{\prime} \in W\left(\sigma^{\prime}\right)$ give the same $w$, then their difference $w_{1}^{\prime}-w_{2}^{\prime}$ is an integral linear combination of $v_{i j}$. However, then $w_{1}^{\prime}-w_{2}^{\prime}$ is also an integral linear combination of $v_{i j}^{\prime}$ because $\mathbb{Z}\left\{v_{i j}^{\prime}\right\}_{i, j}=\mathbb{Z}\left\{v_{i j}\right\}_{i, j} \cap N_{\sigma^{\prime}}$. Hence $w_{1}^{\prime}-w_{2}^{\prime}=0$.

\section{Modified Barycentric Subdivisions}

Let $f_{\Delta}: \Delta_{X} \rightarrow \Delta_{B}$ be a simplicial morphism. Consider the barycentric subdivision $B S\left(\Delta_{B}\right)$ of $\Delta_{B}$. The one-dimensional cones of $B S\left(\Delta_{B}\right)$ are of the form $\mathbb{R}_{+} \hat{\tau}$ where $\hat{\tau}=\sum u_{i}$ is the barycenter of a cone $\tau \in \Delta_{B}$ with primitive points $u_{1}, \ldots, u_{m}$. A cone $\tau^{\prime} \in B S\left(\Delta_{B}\right)$ is spanned by $\hat{\tau}_{1}, \ldots, \hat{\tau}_{k}$, where $\tau_{1} \leq \tau_{2} \leq \cdots \leq \tau_{k}$ is a chain of cones in $\Delta_{B}$

In general, $f_{\Delta}$ does not induce a morphism $B S\left(\Delta_{X}\right) \rightarrow B S\left(\Delta_{B}\right)$. For example, if $\sigma=\left\langle v_{11}, v_{12}, v_{21}\right\rangle, \tau=\left\langle u_{1}, u_{2}\right\rangle$, and $f_{\Delta}: v_{i j} \mapsto u_{i}$, then $f_{\Delta}$ does not induce a morphism of barycentric subdivisions. To get a morphism we need to modify the barycenters $\hat{\sigma}$ of cones $\sigma \in \Delta_{X}$ so that they map to (multiples of) barycenters of $\Delta_{B}$.

Definition 3.1. The data of modified barycenters consists of:

1. A subset of cones $\tilde{\Delta}_{X} \subset \Delta_{X}$.

2. For each cone $\sigma \in \tilde{\Delta}_{X}$ a lattice point $b_{\sigma} \in \operatorname{int}(\sigma) \cap N_{\sigma}$ such that $f_{\Delta}\left(b_{\sigma}\right) \in \mathbb{R}_{+} \hat{\tau}$ for some $\tau \in \Delta_{B}$.

Recall that for any total order $\prec$ on the set of cones in $\Delta_{X}$ refining the partial order $\leq$, the barycentric subdivision $B S\left(\Delta_{X}\right)$ can be realized as a sequence of star subdivisions at the barycenters $\hat{\sigma}$ for all cones $\sigma \in \Delta_{X}$ in the descending order $\prec$.

Definition 3.2. Given modified barycenters $\left(\tilde{\Delta}_{X},\left\{b_{\sigma}\right\}\right)$ and a total order $\prec$ on $\Delta_{X}$ refining the partial order $\leq$, the modified barycentric subdivision $M B S_{\tilde{\Delta}_{X},\left\{b_{\sigma}\right\}, \prec}\left(\Delta_{X}\right)$ is the sequence of star subdivisions at $b_{\sigma}$ for all $\sigma \in \tilde{\Delta}_{X}$ in the descending order $\prec$.

Example 3.3. Let $f_{\Delta}:\left\langle v_{11}, v_{12}, v_{21}\right\rangle \rightarrow\left\langle u_{1}, u_{2}\right\rangle$ be the morphism defined by $f_{\Delta}: v_{i j} \mapsto u_{i}$. Let $\tilde{\Delta}_{X}$ consist of the two cones $\tilde{\Delta}_{X}=\left\{\left\langle v_{11}, v_{21}\right\rangle,\left\langle v_{12}, v_{21}\right\rangle\right\}$, and let the modified barycenters be $\left\{b_{\sigma}\right\}=\left\{v_{11}+v_{21}, v_{12}+v_{21}\right\}$. Depending on whether $\left\langle v_{11}, v_{21}\right\rangle \prec\left\langle v_{12}, v_{21}\right\rangle$ or vice versa, we get two modified barycentric subdivisions as shown in Fig. 1.

To simplify notation, we write $M B S_{\tilde{\Delta}_{X}}\left(\Delta_{X}\right)$ or simply $M B S\left(\Delta_{X}\right)$ instead of $M B S_{\tilde{\Delta}_{X},\left\{b_{\sigma}\right\}, \prec}\left(\Delta_{X}\right)$. By definition, $\operatorname{MBS}\left(\Delta_{X}\right)$ is a projective simplicial subdivision of 

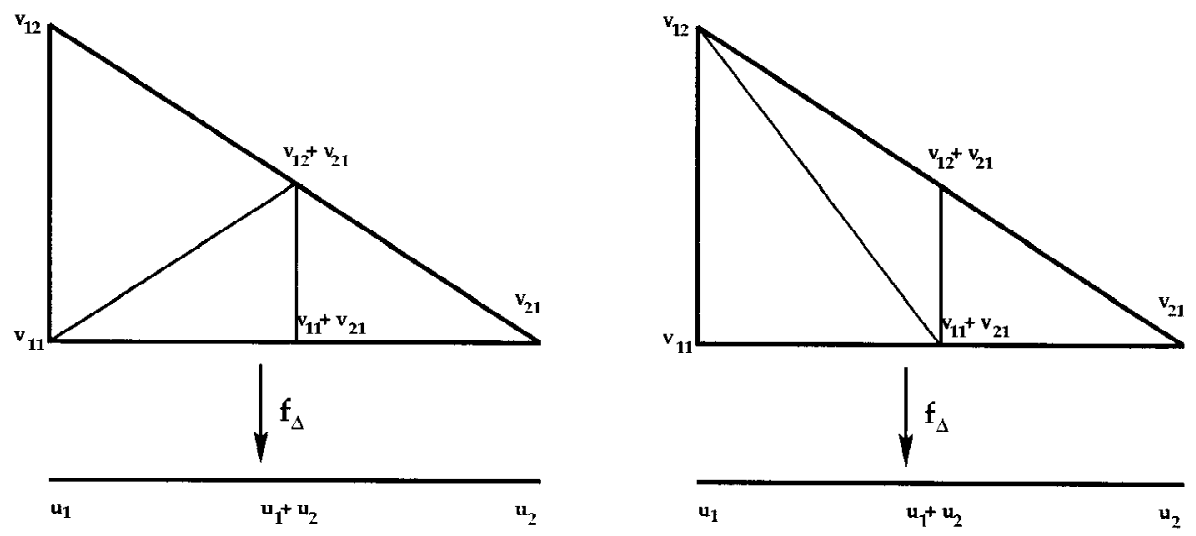

Fig. 1. Two modified barycentric subdivisions from Example 3.3

$\Delta_{X}$. Next, we show that, as in the case of the ordinary barycentric subdivision, the cones of $\operatorname{MBS}\left(\Delta_{X}\right)$ can be characterized by chains of cones in $\Delta_{X}$. We may assume that the zero- and one-dimensional cones of $\Delta_{X}$ are all in $\tilde{\Delta}_{X}$, and they precede all other cones in the order $\prec$. For a cone $\sigma \in \Delta_{X}$ let $\tilde{\sigma}$ be the maximal face of $\sigma$ (with respect to $\prec$ ) in $\tilde{\Delta}_{X}$. Given a chain of cones $\sigma_{1} \leq \cdots \leq \sigma_{k}$ in $\Delta_{X}$, the cone $\left\langle b_{\tilde{\sigma}_{1}}, \ldots, b_{\tilde{\sigma}_{k}}\right\rangle$ is a subcone of $\sigma_{k}$. Let $C\left(\Delta_{X}\right)$ be the set of all such cones corresponding to chains $\sigma_{1} \leq \cdots \leq \sigma_{k}$ in $\Delta_{X}$.

Proposition 3.4. $C\left(\Delta_{X}\right)=\operatorname{MBS}\left(\Delta_{X}\right)$.

Proof. We do induction on the number of cones in $\tilde{\Delta}_{X}$ of dimension at least 2 . If $\tilde{\Delta}_{X}$ contains only zero- or one-dimensional cones, then the statement is trivial. So, assume that $\tilde{\Delta}_{X}=\tilde{\Delta}_{X, 0} \cup\left\{\sigma_{0}\right\}$, where $\sigma \prec \sigma_{0}$ for any $\sigma \in \tilde{\Delta}_{X, 0}$, and assume that the proposition is proved for $\tilde{\Delta}_{X, 0}$.

Without loss of generality we may assume that $\Delta_{X}$ consists of cones containing $\sigma_{0}$ and their faces only. We get $M B S_{\tilde{\Delta}_{X}}\left(\Delta_{X}\right)$ from $\Delta_{X}$ if we first subdivide at $b_{\sigma_{0}}$ and then at $b_{\sigma}$ for $\sigma \in \tilde{\Delta}_{X, 0}$ in the descending order $\prec$. If $\Delta_{X, 0}$ is the subcomplex of $\Delta_{X}$ consisting of cones not containing $\sigma_{0}$, then the star subdivision of $\Delta_{X}$ at $b_{\sigma_{0}}$ is $\Delta_{X, 0} \times\left\langle b_{\sigma_{0}}\right\rangle$. Since $\sigma_{0}$ is greater than any $\sigma \in \tilde{\Delta}_{X, 0}$ with respect to $\prec$, all $b_{\sigma} \in \Delta_{X, 0}$, and we see that

$$
M B S_{\tilde{\Delta}_{X}}\left(\Delta_{X}\right)=M B S_{\tilde{\Delta}_{X, 0}}\left(\Delta_{X, 0}\right) \times\left\langle b_{\sigma_{0}}\right\rangle .
$$

A cone in $M B S_{\tilde{\Delta}_{X, 0}}\left(\Delta_{X, 0}\right) \times\left\langle b_{\sigma_{0}}\right\rangle$ is of the form $\sigma \times \rho$, where $\rho$ is a face of $\left\langle b_{\sigma_{0}}\right\rangle$, i.e. either $\{0\}$ or $\left\langle b_{\sigma_{0}}\right\rangle$ itself, and where $\sigma$ is a cone in $M B S_{\tilde{\Delta}_{X, 0}}\left(\Delta_{X, 0}\right)$. Applying induction hypothesis to $M B S_{\tilde{\Delta}_{X, 0}}\left(\Delta_{X, 0}\right)$, we get that $\sigma=\left\langle b_{\tilde{\sigma}_{1}}, \ldots, b_{\tilde{\sigma}_{l}}\right\rangle$ for a chain of cones $\sigma_{1} \leq$ $\cdots \leq \sigma_{l}$ in $\Delta_{X, 0}$. Now if $\rho=\{0\}$, then $\sigma \times \rho=\left\langle b_{\tilde{\sigma}_{1}}, \ldots, b_{\tilde{\sigma}_{l}}\right\rangle \in C\left(\Delta_{X}\right)$. If $\rho=\left\langle b_{\sigma_{0}}\right\rangle$, we let $\sigma_{l+1}$ be a cone in $\Delta_{X}$ that contains both $\sigma_{l}$ and $\sigma_{0}$. Then $\tilde{\sigma}_{l+1}=\sigma_{0}$, and $\sigma \times \rho=$ $\left\langle b_{\tilde{\sigma}_{1}}, \ldots, b_{\tilde{\sigma}_{l}}, b_{\tilde{\sigma}_{l+1}}\right\rangle \in C\left(\Delta_{X}\right)$.

Conversely, let $\left\langle b_{\tilde{\sigma}_{1}}, \ldots, b_{\tilde{\sigma}_{l}}\right\rangle$ be a cone in $C\left(\Delta_{X}\right)$ for some chain $\sigma_{1} \leq \cdots \leq \sigma_{l}$ in $\Delta_{X}$. Then for some $k \leq l$ we have that $\tilde{\sigma}_{1}, \ldots, \tilde{\sigma}_{k} \in \tilde{\Delta}_{X, 0}$, and $\tilde{\sigma}_{k+1}=\cdots=\tilde{\sigma}_{l}=\sigma_{0}$. 
By induction hypothesis, the cone $\left\langle b_{\tilde{\sigma}_{1}}, \ldots, b_{\tilde{\sigma}_{k}}\right\rangle$ coming from the chain $\sigma_{1} \leq \cdots \leq \sigma_{k}$ in $\Delta_{X, 0}$ is in $M B S_{\tilde{\Delta}_{X, 0}}\left(\Delta_{X, 0}\right)$. Hence the cone $\left\langle b_{\tilde{\sigma}_{1}}, \ldots, b_{\tilde{\sigma}_{l}}\right\rangle$ is of the form $\sigma \times \rho$, where $\sigma=\left\langle b_{\tilde{\sigma}_{1}}, \ldots, b_{\tilde{\sigma}_{k}}\right\rangle \in M B S_{\tilde{\Delta}_{X, 0}}\left(\Delta_{X, 0}\right)$, and $\rho=\left\langle b_{\sigma_{0}}\right\rangle$ if $k<l$, and $\rho=\{0\}$ if $k=l$.

Corollary 3.5. Assume that $f_{\Delta}: \Delta_{X} \rightarrow \Delta_{B}$ is a simplicial morphism. If $f_{\Delta}(\tilde{\sigma})=$ $f_{\Delta}(\sigma)$ for all $\sigma \in \Delta_{X}$, then $f_{\Delta}$ induces a simplicial morphism $f_{\Delta}^{\prime}: \operatorname{MBS}\left(\Delta_{X}\right) \rightarrow$ $B S\left(\Delta_{B}\right)$.

Proof. Let $\sigma^{\prime} \in \operatorname{MBS}\left(\Delta_{X}\right)$ correspond to a chain $\sigma_{1} \leq \cdots \leq \sigma_{k}$ in $\Delta_{X}$. Since $f_{\Delta}$ is simplicial, we have a chain of cones $f_{\Delta}\left(\sigma_{1}\right) \leq \cdots \leq f_{\Delta}\left(\sigma_{k}\right)$ in $\Delta_{B}$. Recall that $b_{\tilde{\sigma}_{i}}$ is mapped to a multiple of a barycenter: $f_{\Delta}\left(b_{\tilde{\sigma}_{i}}\right)=\mathbb{R}_{+} \hat{\tau}$ for some $\tau \in \Delta_{B}$. The assumption that $f_{\Delta}\left(\tilde{\sigma}_{i}\right)=f_{\Delta}\left(\sigma_{i}\right)$ implies that $f_{\Delta}\left(b_{\tilde{\sigma}_{i}}\right) \in \mathbb{R}_{+} \widehat{f_{\Delta}\left(\sigma_{i}\right)}$, hence the cone $\left\langle b_{\tilde{\sigma}_{1}}, \ldots, b_{\tilde{\sigma}_{k}}\right\rangle$ is mapped onto the cone $\left\langle\widehat{f_{\Delta}\left(\sigma_{1}\right)}, \ldots, \widehat{f_{\Delta}\left(\sigma_{k}\right)}\right\rangle \in B S\left(\Delta_{B}\right)$.

Example 3.6. Assume that $f_{\Delta}: \Delta_{X} \rightarrow \Delta_{B}$ is a simplicial morphism taking primitive points of $\Delta_{X}$ to primitive points of $\Delta_{B}$. Then for a cone $\sigma \in \Delta_{X}$ such that $f_{\Delta}: \sigma \stackrel{\simeq}{\rightarrow} \tau$ for some $\tau \in \Delta_{B}$, we have $f_{\Delta}(\hat{\sigma})=\hat{\tau}$.

Let $\bar{\Delta}_{X}=\left\{\sigma \in \Delta_{X}:\left.f_{\Delta}\right|_{\sigma}\right.$ is injective $\}, b_{\sigma}=\hat{\sigma}$. Clearly, the hypothesis of the lemma is satisfied, and we have a simplicial morphism $f_{\Delta}^{\prime}: M B S_{\bar{\Delta}_{X}}\left(\Delta_{X}\right) \rightarrow B S\left(\Delta_{B}\right)$.

Conversely, if $\left(\tilde{\Delta}_{X},\left\{b_{\sigma}\right\}\right)$ is the data of modified barycenters such that $f_{\Delta}$ induces a morphism $f_{\Delta}^{\prime}: M B S_{\tilde{\Delta}_{X}}\left(\Delta_{X}\right) \rightarrow B S\left(\Delta_{B}\right)$, then $\bar{\Delta}_{X} \subset \tilde{\Delta}_{X}$. Thus, we may always assume that $\bar{\Delta}_{X} \subset \tilde{\Delta}_{X}$.

\section{Reducing the Multiplicity of $\Delta_{X}$}

Proposition 4.1. Let $f_{\Delta}: \Delta_{X} \rightarrow \Delta_{B}$ be a simplicial morphism taking primitive points to primitive points. Assume that $\Delta_{B}$ is nonsingular, $\Delta_{X}$ is singular, and every singular cone $\sigma \in \Delta_{X}$ contains a point $w \in W(\sigma) \backslash\{0\}$ mapping to a barycenter in $\Delta_{B}$. Then there exists a modified barycentric subdivision $M B S\left(\Delta_{X}\right)$ of $\Delta_{X}$ having multiplicity strictly less than the multiplicity of $\Delta_{X}$ such that $f_{\Delta}$ induces a simplicial morphism $f_{\Delta}^{\prime}: \operatorname{MBS}\left(\Delta_{X}\right) \rightarrow B S\left(\Delta_{B}\right)$.

Proof. For every singular cone $\sigma \in \Delta_{X}$ we choose a point $w_{\sigma}$ as follows. By assumption, there exists a point $w \in W(\sigma) \backslash\{0\}$ mapping to a barycenter of $\Delta_{B}: f_{\Delta}(w)=\hat{\tau}$. Then for a unique cone $\tau_{0} \in \Delta_{B}$ we have $f_{\Delta}(\sigma)=\tau \times \tau_{0}$. We choose a face $\sigma_{0} \leq \sigma$ such that $f_{\Delta}: \sigma_{0} \stackrel{\simeq}{\rightarrow} \tau_{0}$. Set $w_{\sigma}=w+\hat{\sigma}_{0}$; then

$$
f_{\Delta}\left(w_{\sigma}\right)=f_{\Delta}(w)+f_{\Delta}\left(\hat{\sigma}_{0}\right)=\hat{\tau}+\hat{\tau}_{0}=\widehat{f_{\Delta}(\sigma)} .
$$

Having chosen the set $\left\{w_{\sigma}\right\}$, we may remove some of the points $w_{\sigma}$ if necessary so that every simplex $\rho \in \Delta_{X}$ contains at most one $w_{\sigma}$ in its interior. With $\bar{\Delta}_{X}$ as in Example 3.6, let $\tilde{\Delta}_{X}=\bar{\Delta}_{X} \cup\left\{\rho \in \Delta_{X} \mid w_{\sigma} \in\right.$ int $(\rho)$ for some singular $\left.\sigma\right\}, b_{\rho}=\hat{\rho}$ if $\rho \in \bar{\Delta}_{X}$, and $b_{\rho}=w_{\sigma}$ if $w_{\sigma} \in \operatorname{int}(\rho)$. 
Next we specify the order $\prec$. We refine the partial order $\leq$ as follows: for two faces $\sigma_{1}$ and $\sigma_{2}$ of a cone $\sigma \in \Delta_{X}$ we set $\sigma_{1} \prec_{0} \sigma_{2}$ if $\operatorname{dim} f_{\Delta}\left(\sigma_{1}\right)<\operatorname{dim} f_{\Delta}\left(\sigma_{2}\right)$. Since $\bar{\Delta}_{X} \subset \tilde{\Delta}_{X}$, this ensures that the condition $f_{\Delta}(\tilde{\sigma})=f_{\Delta}(\sigma)$ of Corollary 3.5 is satisfied for any refinement of $\prec_{0}$. Now if $\sigma$ is singular, then the point $w_{\sigma}$ constructed above lies in the interior of a face $\rho_{\sigma}$ such that $f_{\Delta}\left(\rho_{\sigma}\right)=f_{\Delta}(\sigma)$. We further refine the order $\prec_{0}$ by setting $\sigma_{1} \prec_{0} \rho_{\sigma}$ for any face $\sigma_{1}$ of the singular cone $\sigma$. Then $\tilde{\sigma}=\rho_{\sigma}$ whenever $\sigma$ is singular. Finally we let $\prec$ be any refinement of $\prec_{0}$ to a total order.

Let $\sigma \in \Delta_{X}$ be a cone, and let a maximal cone $\sigma^{\prime} \in M B S\left(\Delta_{X}\right)$ be given by a maximal chain of faces of $\sigma: \sigma_{1} \leq \cdots \leq \sigma_{n}$. We have to show that $m\left(\sigma^{\prime}, N_{\sigma^{\prime}}\right) \leq m\left(\sigma, N_{\sigma}\right)$, and if $\sigma$ is singular, then the inequality is strict.

We can order the primitive points $v_{1}, \ldots, v_{n}$ of $\sigma$ so that $\sigma_{1}=\left\langle v_{1}\right\rangle, \sigma_{2}=\left\langle v_{1}, v_{2}\right\rangle, \ldots$, $\sigma_{n}=\left\langle v_{1}, \ldots, v_{n}\right\rangle$. Since $b_{\tilde{\sigma}_{i}} \in \sigma_{i}$, the primitive points of $\sigma^{\prime}=\left\langle b_{\tilde{\sigma}_{1}}, \ldots, b_{\tilde{\sigma}_{n}}\right\rangle$ can be written as

$$
\begin{aligned}
v_{1}^{\prime} & =\frac{1}{\mu_{1}} b_{\tilde{\sigma}_{1}}=a_{11} v_{1}, \\
v_{2}^{\prime} & =\frac{1}{\mu_{2}} b_{\tilde{\sigma}_{2}}=a_{21} v_{1}+a_{22} v_{2}, \\
& \cdots \\
v_{n}^{\prime} & =\frac{1}{\mu_{n}} b_{\tilde{\sigma}_{n}}=a_{n 1} v_{1}+\cdots+a_{n n} v_{n}
\end{aligned}
$$

for some $a_{i j} \geq 0$ and integers $\mu_{i} \geq 1$. The multiplicity of $\sigma^{\prime}$ is $a_{11} \cdot a_{22} \cdots a_{n n}$ times the multiplicity of $\sigma$. By the choice of $b_{\rho}$ above, all $a_{i i} \leq 1$, hence $m\left(\sigma^{\prime}, N_{\sigma^{\prime}}\right) \leq m\left(\sigma, N_{\sigma}\right)$. If $\sigma$ is singular, let $i$ be the smallest index such that the face $\sigma_{i}$ is singular. Then, with notation as above, $b_{\tilde{\sigma}_{i}}=w+\hat{\sigma}_{0}$ for some $w \in W\left(\sigma_{i}\right) \backslash\{0\}$, and $\sigma_{0} \leq \sigma_{i}$. Now if $a_{i i}=1$, then $w \in\left\langle v_{1}, \ldots, v_{i-1}\right\rangle$, and this gives a contradiction with the choice of $i$. Hence $a_{i i}<1$ and $m\left(\sigma^{\prime}, N_{\sigma^{\prime}}\right)<m\left(\sigma, N_{\sigma}\right)$.

\section{Families of Surfaces and Threefolds}

Proof of Theorem 1.6. Let $f_{\Delta}: \Delta_{X} \rightarrow \Delta_{B}$ be a surjective morphism of polyhedral complexes such that $f_{\Delta}^{-1}(0)=\{0\}$. It is shown in [AK] that there exist projective simplicial subdivisions $\Delta_{X}^{\prime}$ of $\Delta_{X}$ and $\Delta_{B}^{\prime}$ of $\Delta_{B}$ such that $\Delta_{B}$ is nonsingular and $f_{\Delta}$ induces a simplicial morphism $f_{\Delta}^{\prime}: \Delta_{X}^{\prime} \rightarrow \Delta_{B}^{\prime}$. To obtain these subdivisions, one first subdivides $\Delta_{B}$ such that the image of every cone in $\Delta_{X}$ is a union of cones in $\Delta_{B}^{\prime}$. The convex piecewise linear function defining the subdivision $\Delta_{B}^{\prime}$ can then be composed with $f_{\Delta}$ to give a subdivision of $\Delta_{X}$. A sequence of star subdivisions centered at the onedimensional edges yields the required simplicial subdivision $\Delta_{X}^{\prime}$. Thus, we may assume that $\Delta_{X}$ is simplicial, $\Delta_{B}$ is nonsingular, and $f_{\Delta}: \Delta_{X} \rightarrow \Delta_{B}$ is a simplicial map.

Applying the construction of [KKMS] over the edges of $\Delta_{B}$ (Section 2.2), we can make $\left.f_{\Delta}\right|_{\Delta_{X, i}}$ semistable without increasing the multiplicity of $\Delta_{X}$. We show below that every singular simplex $\sigma \in \Delta_{X}$ contains a point $w \in W(\sigma) \backslash\{0\}$ mapping to a barycenter of $\Delta_{B}$. By Proposition 4.1, there exists a modified barycentric subdivision such that $f_{\Delta}$ induces a simplicial morphism $f_{\Delta}^{\prime}: M B S\left(\Delta_{X}\right) \rightarrow B S\left(\Delta_{B}\right)$, with multiplicity of 
$\operatorname{MBS}\left(\Delta_{X}\right)$ strictly less than the multiplicity of $\Delta_{X}$. Since $f_{\Delta}^{\prime}$ is simplicial and $B S\left(\Delta_{B}\right)$ nonsingular, the proof is completed by induction on the multiplicity of $\Delta_{X}$.

Consider the restriction of $f_{\Delta}$ to a singular simplex $f_{\Delta}: \sigma \rightarrow \tau$, where $\tau$ has primitive points $u_{1}, \ldots, u_{m}, \sigma$ has primitive points $v_{i j}, i=1, \ldots, m, j=1, \ldots, J_{i}$, and $f_{\Delta}\left(v_{i j}\right)=u_{i}$. Since $\sigma$ is singular, it contains a point $w \in W(\sigma) \backslash\{0\}$ :

$$
w=\sum_{i, j} \alpha_{i j} v_{i j}, \quad 0 \leq \alpha_{i j}<1, \quad \sum \alpha_{i j}>0 .
$$

Considering a face of $\sigma$ if necessary, we may assume that $w$ lies in the interior of $\sigma$, hence $0<\alpha_{i j}$. Since $f_{\Delta}(w) \in N_{\tau}$, it follows that $\sum_{j} \alpha_{i j} \in \mathbb{Z}$ for all $i$. In particular, if $J_{i_{0}}=1$ for some $i_{0}$, then $\alpha_{i_{0}}=0$, and $w$ lies in a face of $\sigma$. So we may assume that $J_{i}>1$ for all $i$. Since the relative dimension of $f_{\Delta}$ is $\sum_{i}\left(J_{i}-1\right)$, we have to consider all possible decompositions $\sum_{i}\left(J_{i}-1\right) \leq 3$, where $J_{i}>1$ for all $i$.

The cases when the relative dimension of $f_{\Delta}$ is 0 or 1 are trivial and left to the reader.

If the relative dimension of $f_{\Delta}$ is 2 , then either $J_{1}=3$ or $J_{1}=J_{2}=2$. In the first case we have that $\left\langle v_{11}, v_{12}, v_{13}\right\rangle$ is singular, contradicting the semistability of $\left.f_{\Delta}\right|_{\Delta_{X, 1}}$. In the second case, $\alpha_{11}+\alpha_{12}, \alpha_{21}+\alpha_{22} \in \mathbb{Z}$ and $0<\alpha_{i j}<1$ imply that $\alpha_{11}+\alpha_{12}=$ $\alpha_{21}+\alpha_{22}=1$. Hence $f_{\Delta}(w)=u_{1}+u_{2}$ is a barycenter.

In relative dimension 3 , either $J_{1}=4$, or $J_{1}=3, J_{2}=2$, or $J_{1}=J_{2}=J_{3}=2$. In the first case we get a contradiction with the semistability of $\left.f_{\Delta}\right|_{\Delta_{X, 1}}$; the third case gives $\alpha_{11}+\alpha_{12}=\alpha_{21}+\alpha_{22}=\alpha_{31}+\alpha_{32}=1$ as for relative dimension 2 . In the second case, either $\alpha_{11}+\alpha_{12}+\alpha_{13}=\alpha_{21}+\alpha_{22}=1$ and $w$ maps to a barycenter, or $\alpha_{11}+\alpha_{12}+\alpha_{13}=2, \alpha_{21}+\alpha_{22}=1$ and $\left(\sum v_{i j}\right)-w$ maps to a barycenter.

Example 5.1. We show by an example that the previous construction of modified barycentric subdivisions does not work in relative dimension $\geq 4$. Let $\tau=\left\langle u_{1}, u_{2}\right\rangle$ and $\sigma=\left\langle v_{11}, v_{12}, v_{13}, v_{14}, v_{21}, v_{22}\right\rangle$, with lattices $N_{\tau}=\mathbb{Z}\left\{u_{1}, u_{2}\right\}$ and $N_{\sigma}=\mathbb{Z}\left\{v_{11}, \ldots, v_{22}\right.$, $\left.\frac{1}{2}\left(v_{11}+\cdots+v_{22}\right)\right\}$. Then $W(\sigma) \backslash\{0\}$ consists of a single point $w=\frac{1}{2}\left(v_{11}+\cdots+v_{22}\right)$, and if $f_{\Delta}$ maps $v_{i j}$ to $u_{i}$, then $w$ is mapped to $2 u_{1}+u_{2}$, which is not a barycenter.

\section{Acknowledgments}

The suggestion to write up the proof of semistable reduction for low relative dimensions came from Dan Abramovich. I also wish to thank the anonymous reviewers for useful suggestions.

\section{References}

[AK] D. Abramovich and K. Karu, Weak semistable reduction in characteristic 0, preprint. alggeom/9707012.

[dJ] A. J. de Jong, Smoothness, semistability, and alterations, Publ. Math. I.H.E.S. 83 (1996), 51-93.

[F] W. Fulton, Introduction to Toric Varieties, Princeton University Press, Princeton, NJ, 1993.

[KKMS] G. Kempf, F. Knudsen, D. Mumford, and B. Saint-Donat, Toroidal Embeddings I, LNM 339, Springer-Verlag, Berlin, 1973. 